\title{
Physics-Based Modeling of Aortic Wall Motion from ECG-Gated 4D Computed Tomography
}

\author{
Guanglei Xiong $^{1}$ and Charles A. Taylor ${ }^{2}$ \\ ${ }^{1}$ Biomedical Informatics Program, Stanford University, CA, USA \\ ${ }^{2}$ Departments of Bioengineering and Surgery, Stanford University, CA, USA \\ \{glxiong, taylorca\} astanford.edu
}

\begin{abstract}
Recent advances in electrocardiogram (ECG)-gated Computed Tomography (CT) technology provide $4 \mathrm{D}(3 \mathrm{D}+\mathrm{T})$ information of aortic wall motion in high spatial and temporal resolution. However, imaging artifacts, e.g. noise, partial volume effect, misregistration and/or motion blurring may preclude its usability in many applications where accuracy and reliability are concerns. Although it is possible to find correspondence through tagged MRI or echo or image registration, it may be either inconsistent to the physics or difficult to utilize data from all frames. In this paper, we propose a physics-based filtering approach to construct a dynamic model from these 4D images. It includes a state filter that corrects simulated displacements from an elastic finite element model to match observed motion from images. In the meantime, the model parameters are refined to improve the model quality by applying a parameter filter based on ensemble Kalman filtering. We evaluated the performance of our method on synthetic data where ground-truths are available. Finally, we successfully applied the method to a real data set.
\end{abstract}

Keywords: physics-based modeling, dynamic model, aorta, wall motion, Kalman filtering, 4DCT.

\section{Introduction}

CT angiography (CTA) is now considered as a preferred technique to conventional Xray angiogram for assessing of diseases of heart, coronary artery, and thoracic aorta [1]. The development of CTA with ECG gating has been driven by imaging of coronary artery but it is also quite beneficial to the imaging of the thoracic aorta [2]. Although the frame rate has not yet reached typical video rates, it is already adequate to characterize the aortic wall motion. Visualization using real-time rendering has been routinely used to qualitatively examine functional abnormalities of the aorta that are not obvious on static images. In contrast, only a limited number of preliminary quantitative studies were carried out in healthy [3] or disease-related cases [4].

Although these studies have provided useful insights of aortic wall motion, there are several drawbacks that need to be improved, which is the purpose of this paper. First, imaging artifacts, such as noise, partial volume effect, misregistration, and/or motion blurring, should be explicitly considered since the goal is to quantify the motion. A physics-based approach may be helpful to overcome the effects of these artifacts, because the 
motion of the aorta should obey general principles of elasticity. Another advantage of this approach is the ability to obtain not only kinematic quantities, e.g. strain, but also the dynamic quantities, e.g. stress. Second, all data, both spatial and temporal, should be utilized in the analysis. It is beneficial to make use of full volumes of images instead of 2D slices and all frames in a cycle rather than merely two single systolic and diastolic phases. Third, it is helpful to establish correspondences or trajectories of material points across all frames. Although tagged MRI or echo or image registration is traditionally used for this task, the problems are inconsistency to the physics and difficulty to utilize data from all frames. A compromise between the image data and the physical model may be necessary in this respect. Lastly, the model could be improved by refining its parameters, i.e. the physical properties of the wall. This step is necessary and important because the dynamic model only becomes valid and useful once the parameters are correct. In other words, the model with reasonable parameters explains better what are observed in images.

A relevant area is to extract heart (or mostly left ventricle) motion in cardiac-gated SPECT or CT. Most recently, temporal relationship is explicitly employed in some studies. To ensure robustness of the segmentation, temporally smoothing regularization in deformable models is naturally taken into account $[5,6]$. However, these models are empirical, not necessarily physical. Until recently, Moireau et al. proposed filtering techniques to estimate heart motion from 4D position measurements [7], although results were only demonstrated on synthetic data. In contrast of heart motion, studies of quantification of thoracic aorta motion are lacking. While pulsation in the thoracic aorta is not as large as that in the heart, the cyclic strain is still quite significant, up to $10 \%$ [8], which may have critical implications on surgical interventions, e.g. endovascular aneurysm repair [4]. Therefore, it is demanding of a systematic method to model dynamics of thoracic aorta with the goal of facilitating quantification of thoracic aorta motion. Instead of using image registration approaches, we seek a physics-based filtering approach for this task.

\section{Segmentation and Surface Mesh}

Figure 1 shows a volume-rendered data set of healthy thoracic aorta from ECG-gated 4D CT, which has 10 frames per cardiac cycle. The size for each frame is $512 \times 512 \times 482$. The spatial resolution is $0.62 \times 0.62 \times 1.0 \mathrm{~mm}$. The anatomy of interest includes ascending thoracic aorta, aortic arch, descending thoracic aorta, as well as three main branches: brachiocephalic trunk, left common carotid artery, and left subclavian artery. Prominent motion artifacts are observed in systolic phase (frames 0-3), especially at ascending aorta.

To consider thoracic aorta itself, we need to separate it from other objects, e.g. heart, veins, and bones, etc. We chose to segment thoracic aorta for each frame (shown in Fig. 2) by geodesic active contour method [9]. Only the first frame needs to be segmented from scratch. Segmentations of the following frames are simpler if initialized by that of the last frame noting the difference between neighboring frames is relatively small. 


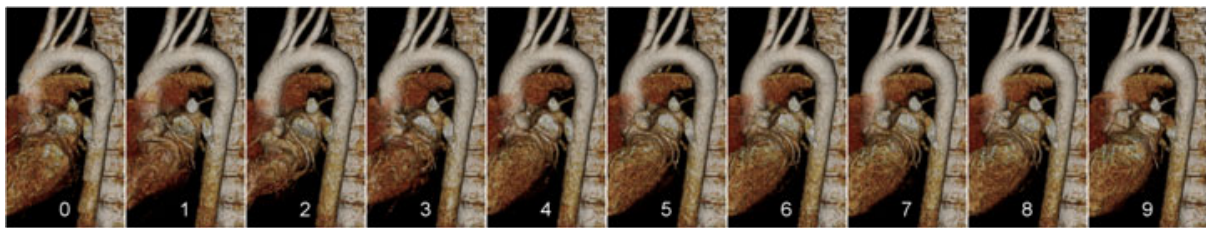

Fig. 1. A volume-rendered data set of thoracic aorta from ECG-gated 4D CT
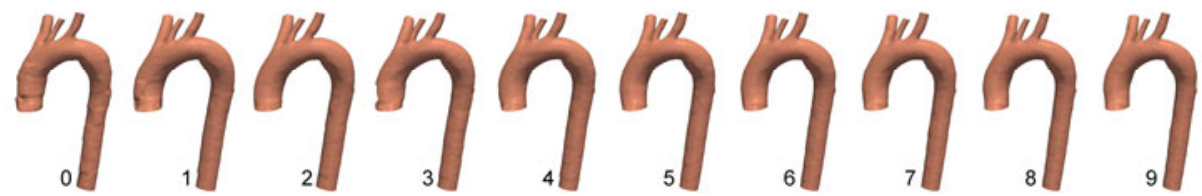

Fig. 2. Segmentations of thoracic aorta in Fig. 1

Since the end-diastole frame (frame 9) often has least noise, the segmentation of this frame is used to extract the triangular mesh (nodes: 1622, triangles: 3144) of the aorta as in Fig. 3(a), upon which will be solved by the finite element method. The mesh is trimmed perpendicular to the vessel wall at all the outlets to get planar boundaries. Note we also include three main branches coming off the aorta arch in the geometric model in Fig. 3(b).
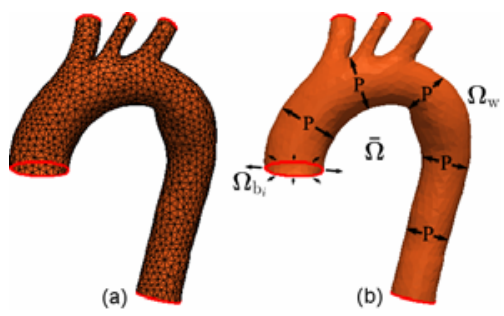

Fig. 3. Surface mesh (a) and geometric model (b)

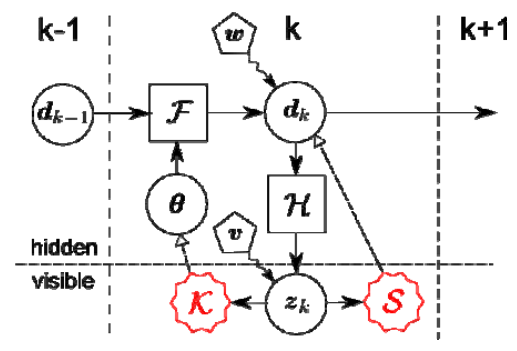

Fig. 4. Overview of our approach (details in text)

\section{Methods}

Figure 4 provides a graphical overview of the proposed physics-based filtering approach to construct a dynamic model of thoracic aorta. The dynamics of the aorta is modeled by an operator $\mathcal{F}$ (defined in Section 3.1), which relates the displacements $\boldsymbol{d}$ (named as states in the sequel) at current time step $\mathrm{k}$ from $\mathrm{k}-1$, with the mechanical properties $\boldsymbol{\theta}$, i.e. Young's modulus (named as parameters), and some additive model error $\boldsymbol{w}$ :

$$
\boldsymbol{d}_{k}=\mathcal{F}\left(\boldsymbol{d}_{k-1}, \boldsymbol{\theta}\right)+\boldsymbol{w}
$$

The observed displacements $\boldsymbol{z}$ (named as measurements) are led by an operator $\mathcal{H}$, from the actual displacements $\boldsymbol{d}$, subject to some measurement error $\boldsymbol{v}$ : 


$$
\boldsymbol{z}_{k}=\mathcal{H}\left(\boldsymbol{d}_{k}\right)+\boldsymbol{v}
$$

Our goals are

(a) To develop a state filter $\mathcal{S}$, which corrects simulated displacements with measurements (Section 3.2):

$$
\hat{\boldsymbol{d}}_{k}=\mathcal{S}\left(\boldsymbol{d}_{k}, \boldsymbol{\theta}, \boldsymbol{z}_{k}\right)
$$

(b) To develop a parameter filter $\mathcal{K}$, which improves the model by refining the parameters (Section 3.3):

$$
\hat{\boldsymbol{\theta}}=\mathcal{K}\left(\boldsymbol{d}_{k}, \boldsymbol{\theta}, \boldsymbol{z}_{k}\right)
$$

Note we separate entities hidden from visible in Fig. 4.

\subsection{Physical Model}

The dynamics of aorta is modeled as a linear elastic membrane. The linearity is justified by the aortic deformation is generally less than $10 \%$ and we do not account for the visco-elastic effects for our scope. Let $\bar{\Omega}=\Omega_{\mathrm{w}} \cup \Omega_{\mathrm{b}_{1}} \cup \Omega_{\mathrm{b}_{2}} \cup \cdots \cup \Omega_{\mathrm{b}_{n}}$ be the entire aorta as in Fig. 3(b), where $\Omega_{\mathrm{w}}$ is the aortic wall and $\boldsymbol{\Omega}_{\mathrm{b}_{i}}$ is the $i$-th boundary contour. The displacement $\boldsymbol{u}=\left[u_{x}, u_{y}, u_{z}\right]$ satisfies:

$$
\rho \ddot{\boldsymbol{u}}=\nabla \cdot \boldsymbol{\sigma}+\boldsymbol{f}
$$

where the stress $\sigma=\left[\sigma_{x x}, \sigma_{y y}, \tau_{x y}, \tau_{x z}, \tau_{y z}\right]$ is [10]:

$$
\frac{E}{1-\nu^{2}}\left[\begin{array}{ccccc}
1 & \nu & 0 & 0 & 0 \\
\nu & 1 & 0 & 0 & 0 \\
0 & 0 & \frac{1-\nu}{2} & 0 & 0 \\
0 & 0 & 0 & \frac{5(1-\nu)}{12} & 0 \\
0 & 0 & 0 & 0 & \frac{5(1-\nu)}{12}
\end{array}\right]\left[\begin{array}{c}
\frac{\partial u_{x}}{\partial x} \\
\frac{\partial u_{y}}{\partial y} \\
\frac{\partial u_{x}}{\partial y}+\frac{\partial u_{y}}{\partial x} \\
\frac{\partial u_{z}}{\partial x} \\
\frac{\partial u_{z}}{\partial y}
\end{array}\right]
$$

where $E$ is Young's modulus and $\nu=0.5$ is the Poisson's ratio. The body force $f$ is related to driving internal pressure (see Fig. 3(b)) and is periodic.

Using finite element method [10], equation (5) reduces to the matrix form:

$$
\boldsymbol{M} \ddot{\boldsymbol{d}}(t)+\boldsymbol{C} \dot{\boldsymbol{d}}(t)+\boldsymbol{K} \boldsymbol{d}(t)=\boldsymbol{F}(t)
$$

where $\boldsymbol{M}, \boldsymbol{C}$, and $\boldsymbol{K}$ are mass, damping, and stiffness matrices, respectively. To eliminate acceleration $\ddot{\boldsymbol{d}}$ and velocity $\dot{\boldsymbol{d}}$, we use a two-step Newmark's time stepping method [11] to solve for displacements $\boldsymbol{d}$, which leads to the operator $\mathcal{F}$ in Eq. (1):

$$
\begin{gathered}
{\left[\boldsymbol{M}+\gamma \Delta t \boldsymbol{C}+\beta \Delta t^{2} \boldsymbol{K}\right] \boldsymbol{d}_{k+1}+\left[-2 \boldsymbol{M}+(1-2 \gamma) \Delta t \boldsymbol{C}+\left(\frac{1}{2}-2 \beta+\gamma\right) \Delta t^{2} \boldsymbol{K}\right] \boldsymbol{d}_{k}} \\
+\left[\boldsymbol{M}-(1-\gamma) \Delta t \boldsymbol{C}+\left(\frac{1}{2}+\beta-\gamma\right) \Delta t^{2} \boldsymbol{K}\right] \boldsymbol{d}_{k-1}=\Delta t^{2}\left[\beta \boldsymbol{F}_{k+1}+\left(\frac{1}{2}-2 \beta+\gamma\right) \boldsymbol{F}_{k}+\left(\frac{1}{2}+\beta-\gamma\right) \boldsymbol{F}_{k-1}\right]
\end{gathered}
$$

where $\Delta t$ is the time step size. $\beta=0.25$ and $\gamma=0.5$ retain second-order accuracy and unconditional stability. For the nodes on the boundary contours $\boldsymbol{\Omega}_{\mathrm{b}_{i}}$, we allow them to deform freely in the radial direction, while keeping them fixed longitudinally by 
specifying Dirichlet boundary condition $\boldsymbol{u} \cdot \boldsymbol{n}_{\mathrm{b}_{i}}=0$ for nodes on $\boldsymbol{\Omega}_{\mathrm{b}_{i}}$ in Eq. (5), where $\boldsymbol{n}_{\mathrm{b}_{i}}$ is the normal of the corresponding trimming plane. The operator $\mathcal{H}$ in Eq. (2) reduces to an observation matrix $\boldsymbol{H}$ where its element is 1 if the corresponding displacement component has measurement, otherwise 0 .

\subsection{State Filter}

To correct simulated displacements from the physical model, we propose to use a state filter to weigh the trajectories between the model and the measurement as shown in Fig. 5. Our approach is justified from the fact that either the model or the measurement is not perfect, i.e. subject to errors as discussed before.

We define a joint strain energy $\Pi(\overline{\boldsymbol{d}})$ from both contributions:

$$
\underbrace{(\overline{\boldsymbol{d}}-\boldsymbol{d})^{T} \boldsymbol{K}(\overline{\boldsymbol{d}}-\boldsymbol{d})}_{\text {from model }}+\varepsilon \underbrace{(\boldsymbol{z}-\boldsymbol{H} \overline{\boldsymbol{d}})^{T} \boldsymbol{W}_{v \boldsymbol{v}}^{-1}(\boldsymbol{z}-\boldsymbol{H} \overline{\boldsymbol{d}})}_{\text {from measurement }}
$$

where $\varepsilon$ controls the relative weighting between model and measurement. Clearly, small $\varepsilon$ pulls the trajectory closer to the model, while large $\varepsilon$ favors measurement. The matrix $\boldsymbol{W}_{v \boldsymbol{v}}$ is the covariance of the measurement errors, which is $\operatorname{var}(\boldsymbol{v}) \boldsymbol{I}$ if they are the same. The filtered trajectory is then $\hat{\boldsymbol{d}}$ that minimizes $\Pi(\overline{\boldsymbol{d}})$, i.e. $\hat{\boldsymbol{d}}=\operatorname{argmin}_{\bar{d}} \Pi(\overline{\boldsymbol{d}})$. A simple differentiation leads to:

$$
\hat{\boldsymbol{d}}=\left(\boldsymbol{K}+\varepsilon \boldsymbol{H}^{T} \boldsymbol{W}_{v v}^{-1} \boldsymbol{H}\right)^{-1}\left(\boldsymbol{K} \boldsymbol{d}+\varepsilon \boldsymbol{H}^{T} \boldsymbol{W}_{v v}^{-1} \boldsymbol{z}\right)
$$

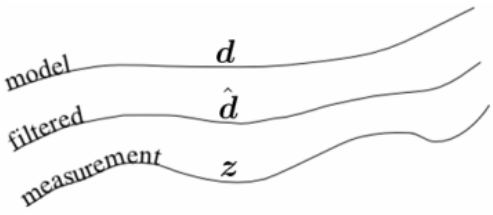

Fig. 5. The state filter weighs between model and measurement

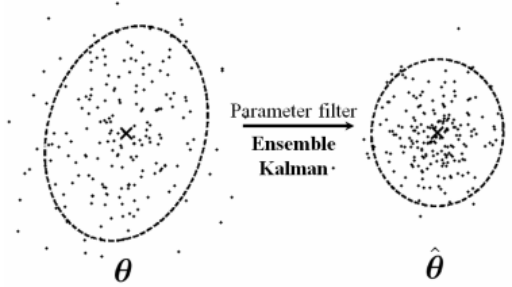

Fig. 6. The parameter filter based on ensemble Kalman filtering

\subsection{Parameter Filter}

To improve the physical model, we employ ensemble Kalman filtering [12] (EnKF) to refine the parameters. EnKF was originated from well-known Kalman filter but specialized for solving large problems as in our problem. It approximates probability densities of system variables as ensembles, i.e. collections of particles (Fig. 6).

In our case, an ensemble for displacements or states $\boldsymbol{D}=\left[\boldsymbol{d}^{1}, \boldsymbol{d}^{2}, \ldots, \boldsymbol{d}^{N}\right]$ and an ensemble for parameters $\Theta=\left[\boldsymbol{\theta}^{1}, \boldsymbol{\theta}^{2}, \ldots, \boldsymbol{\theta}^{N}\right]$ are defined, where $N$ is the number of particles. In general, $N$ should be large enough to represent the underlying distribution. We choose $N=64$ considering the computational cost. Also by subtracting the 
means, we have the state and parameter perturbations: $\widetilde{D}=D-\sum_{n=1}^{N} d_{n} \mathbf{1}_{N}$ and $\widetilde{\boldsymbol{\Theta}}=\boldsymbol{\Theta}-\sum_{n=1}^{N} \boldsymbol{\theta}_{n} \mathbf{1}_{N}$, where $\mathbf{1}_{N}$ is a matrix with all elements equal to $1 / N$. The measurement ensemble is $\boldsymbol{Z}=\left[\boldsymbol{z}^{1}, \boldsymbol{z}^{2}, \ldots, \boldsymbol{z}^{N}\right]$, where each particle $\boldsymbol{z}^{n}=\boldsymbol{z}+\boldsymbol{v}^{n}$ is generated by perturbing random variables $\boldsymbol{v}^{n}$ sampled from the distribution of measurement error $\boldsymbol{v}$. Using the Kalman gain

$$
\boldsymbol{K}=(\boldsymbol{H} \widetilde{\boldsymbol{D}})^{T}\left[(\boldsymbol{H} \widetilde{\boldsymbol{D}})(\boldsymbol{H} \widetilde{\boldsymbol{D}})^{T}+(N-1) \boldsymbol{W}_{\boldsymbol{v} v}\right]^{-1}
$$

The refined parameter ensemble can be written as

$$
\widehat{\Theta}=\Theta+\widetilde{\Theta} \boldsymbol{K}(\boldsymbol{Z}-\boldsymbol{H} \boldsymbol{D})
$$

Finally, the filtered parameter $\hat{\boldsymbol{\theta}}$ is the ensemble mean of $\widehat{\boldsymbol{\Theta}}$. The uncertainty of $\hat{\boldsymbol{\theta}}$ can be estimated by calculating the ensemble variance of $\widehat{\Theta}$. The uncertainty generally decreases after applying the parameter filter as in Fig. 6.

\section{Results}

\subsection{Synthetic Data}

The geometric model was divided into 8 regions, 5 of which are on the aortic wall. Each region was assigned different Young's modulus as shown in Fig.7. The synthetic data were generated with this set of parameters by simulating the dynamics using Eq. (8). The time step size is $1 / 200$ of the cardiac cycle, which is $1.0 \mathrm{~s}$. The model and measurement errors are Gaussian with standard deviations $0.4 \mathrm{~mm}$ and $0.5 \mathrm{~mm}$, respectively.

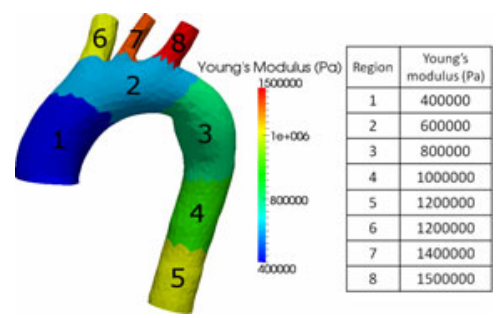

Fig. 7. Division of the geometric model. Each region has a different Young's modulus

The state filter was applied to correct the displacements using measurement. Note the measured displacements were subject to measurement error and the true displacements were blinded to the filter. Figure 8 presents the mean distances from the filtered model to the true model and the effects of different weighting $\varepsilon$. The distance for the non-filtered model is served as the baseline. As $\varepsilon$ increases, the filtered model gets closer to the true model. But it cannot coincide with the true model considering the measurement error. The distance becomes flat after a short period.

Next, we consider refine the parameters for 5 regions on the aortic wall. The parameter ensembles were assigned by randomly sampling from a uniform distribution with minimum $0.5 \times 10^{6}$ and maximum $2.0 \times 10^{6}$. The parameter filter was applied while using state filter with $\varepsilon=10^{3}$. This weighting was selected due to the fact that we need to leave enough differences between simulated displacements and measurements in Eq. (12) to update parameters while correcting the displacement. This is justified because the parameter refinement will make the model trajectory closer to the measurement. 


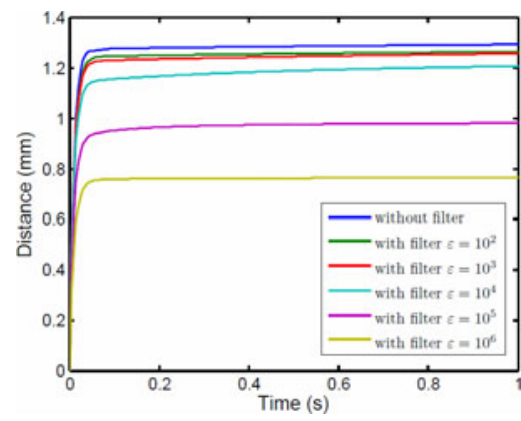

Fig. 8. Mean distance of the filtered model to the true model

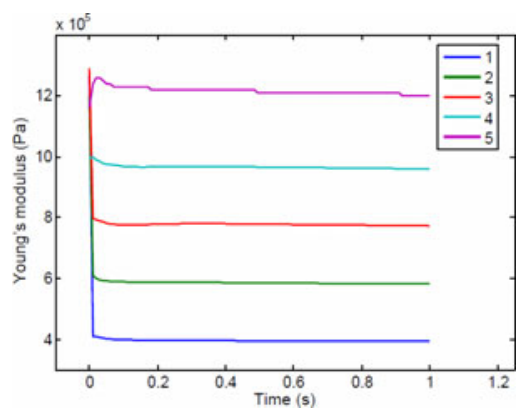

Fig. 9. Parameter refinement on synthetic data

Figure 9 shows the parameter refinement process. We can see that the parameter filter was very efficient to push the parameters to correct values, in less than $1 / 5$ of the cardiac cycle.

\subsection{Real Data}

We consider the real data in Fig. 1. Results with and without the state filter are shown in Fig. 10. The non-filtered result is just from the segmentation where we notice the irregular trajectory of mesh nodes. For the filtered case, the model and measurement

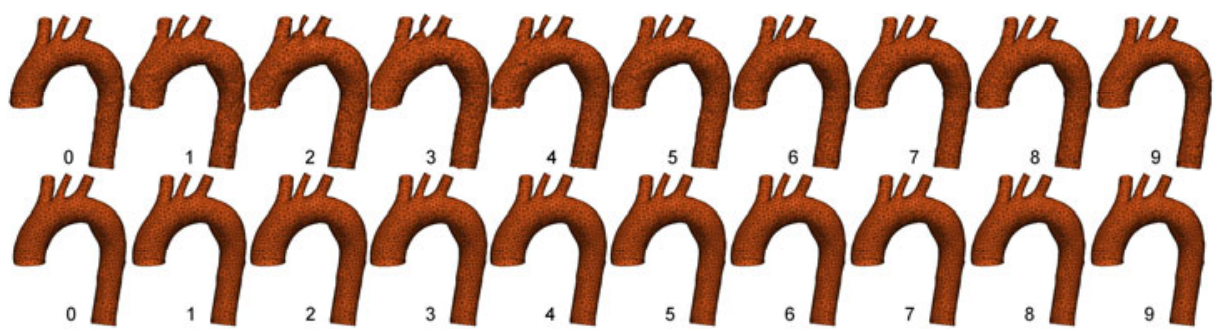

Fig. 10. Results on real data without state filter (top) and with state filter (bottom)

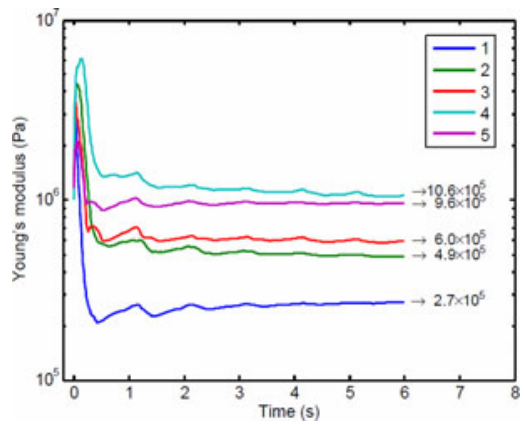

Fig. 11. Parameter refinement on real data 
errors are set to be $0.1 \mathrm{~mm}$ and $0.5 \mathrm{~mm}$. The weighting $\varepsilon$ is $10^{3}$. After the state filter, the trajectories are much smoother. More results with other weightings are provided in supplementary materials. Next, parameter was refined (as shown in Fig. 11) using the proposed parameter filter with the same divisions of the geometric model in Fig. 7. We arrive at a set of meaningful parameters: "distal is stiffer than proximal".

\section{Discussion and Future Work}

We have proposed physics-based filtering techniques that can be used to model the dynamics of thoracic aorta from ECG-gated 4D CT data. The proposed approach features physical model of thoracic aorta, a state filter to correct simulated displacements, and a parameter filter to refine the model parameters. We have tested our method on the synthetic data where the ground truth is available and successfully applied it to a real data set with no known parameters. Our results demonstrate that the approach may be useful to obtain subject-specific kinematic information from 4D $\mathrm{CT}$ and to quantify the dynamics of the thoracic aorta. We believe it is generalizable to other situation involved in $4 \mathrm{D}$ data.

In the future, we will apply the method to more real data sets, especially to some diseased cases, e.g. aneurysms where the wall stiffens. In addition, since we are using a temporally varying but spatially uniform pressure internal to the aortic wall, minor pressure loss and shear force are not currently taken into account. A possibility is to substitute a more sophisticated model of fluid structure interaction. However, this approach needs to be justified since the computational cost dramatically increases and may make parameter filtering intractable in practice. Finally, the parameters obtained on the real data need to be validated before becoming more physically meaningful.

\section{Acknowledgements}

We are grateful to Nan Xiao, Dr. Philippe Moireau and Dr. C. Alberto Figueroa for helpful discussions.

\section{References}

1. Rubin, G.D.: Helical CT Angiography of the Thoracic Aorta. Journal of Thoracic Imaging 12, 128-149 (1997)

2. Marten, K., Funke, M., Rummeny, E.J., Engelke, C.: Electrocardiographic Assistance in Multidetector CT of Thoracic Disorders. Clinical Radiology 60, 8-21 (2005)

3. Lin, F.Y., Devereux, R.B., Roman, M.J., Meng, J., Jow, V.M., Jacobs, A., Weinsaft, J.W., Shaw, L.J., Berman, D.S., Gilmore, A., Callister, T.Q., Min, J.K.: Assessment of the Thoracic Aorta by Multidetector Computed Tomography: Age- and Sex-Specific Reference Values in Adults without Evident Cardiovascular Disease. Journal of Cardiovascular Computed Tomography 2, 298-308 (2008)

4. van Prehn, J., Bartels, L.W., Mestres, G., Vincken, K.L., Prokop, M., Verhagen, H.J., Moll, F.L., van Herwaarden, J.A.: Dynamic Aortic Changes in Patients with Thoracic Aortic Aneurysms Evaluated with Electrocardiography-Triggered Computed Tomographic Angiography before and after Thoracic Endovascular Aneurysm Repair: Preliminary Results. Annals of Vascular Surgery 23, 291-297 (2009) 
5. Montagnat, J., Delingette, H.: 4D Deformable Models with Temporal Constraints: Application to 4D Cardiac Image Segmentation. Medical Image Analysis 9, 87-100 (2005)

6. Brankov, J.G., Yang, Y., Wernick, M.N.: Spatiotemporal Processing of Gated Cardiac Spect Images Using Deformable Mesh Modeling. Medical Physics 32, 2839-2849 (2005)

7. Moireau, P., Chapelle, D., Le Tallec, P.: Filtering for Distributed Mechanical Systems Using Position Measurements: Perspectives in Medical Imaging. Inverse Problems 25, 035010 (2009)

8. Morrison, T.M., Choi, G., Zarins, C.K., Taylor, C.A.: Circumferential and Longitudinal Cyclic Strain of the Human Thoracic Aorta: Age-Related Changes. Journal of Vascular Surgery 49, 1029-1036 (2009)

9. Caselles, V., Kimmel, R., Sapiro, G.: Geodesic Active Contours. International Journal of Computer Vision 22, 61-79 (1997)

10. Hughes, T.J.R.: The Finite Element Method: Linear Static and Dynamic Finite Element Analysis. Prentice-Hall, Englewood Cliffs (1987)

11. Zienkiewicz, O.C.: New Look at Newmark, Houbolt and Other Time Stepping Formulas Weighted Residual Approach. Earthquake Engineering \& Structural Dynamics 5, 413-418 (1977)

12. Evensen, G.: Data Assimilation: The Ensemble Kalman Filter. Springer, New York (2009) 